\title{
Design of Exercises and Test Items for Internetworking Based on a Framework of Exercise Classes
}

\author{
Stefan Freischlad \\ Didactics of Informatics and E-learning, Universität Siegen \\ Hölderlinstraße 3, 57068 Siegen, Germany \\ freischlad@die.informatik.uni-siegen.de
}

\begin{abstract}
Student teachers and teacher novices need an aid to design high quality exercises and test items for Internetworking. In this paper the design of exercises respectively test items is described based on a framework of exercise classes for Internetworking. This framework is composed of content, process, and representation dimension. The content dimension is based on exercise classes for Internetworking and knowledge categories. The process dimension is based on the cognitive process which is needed to solve an exercise and therefore directly influences formulating the question. The third dimension is based on modes of representation and affects the design of the context. The framework was evaluated during a case study in secondary education.
\end{abstract}

\section{Toward High Quality Exercises and Test Items}

Informatics education has to face the difficulties associated with the use of the Internet. International curricula for informatics education describe selected contents $[1,2,3]$ in this field. But there is still no concept for classroom practice. This paper is part of a research project which is promoted by the German Research Foundation (DFG). The aim is to develop and prove a didactic concept, i.e. to describe a concept by means of three components of the Didactic System "Internetworking"1 : Knowledge Networks, Exercise Classes, and Learning Aids. Exercise classes defining and structuring informatics concepts in the field of Internetworking shall support teachers especially novices like teacher students to create or adapt exercises and test items. But we found that there is a gap between applying exercise classes and the design of exercises which is hard to overcome by teacher students. Thus, a concept is needed

${ }^{1}$ The term Internetworking is made up of internetwork and networking. Referring to Merriam-

Webster (http://www.webster.com) the author understands networking as the establishment and use of internetworks. 
which provides teacher students with a framework for the creation of new and for adaptation of existing exercises. This framework will lead to a strategy for the design of exercises. Brinda proposed an approach for a framework in the field of objectoriented modelling [4]. It is derived from an analysis of exercises in this field and is based on a catalogue of identified exercise types which combine several attributes of exercises. An important requirement of the framework which is presented in this paper is to enable teachers to combine several attributes without the inherent restrictions of predefined exercise types.

The overall research project started in 2005 . The author started analysing characteristics of informatics systems based on the Internet to identify necessary competences for using Internet applications [3] and described the theoretical approach towards the components of the Didactic System "Internetworking”. Learning material was developed based on the Didactic System and implemented into classroom practice. After these two projects exercise classes for Internetworking were defined based on the analysis of textbooks. This classification was applied to existing items [5]. In the third project phase these exercise classes were used in teacher education. Teacher students had to design test items during the practical training in school. This lead to the refinement of the framework for exercise classes which is described in this paper. It was used for the design of a test which was performed during the third classroom project. The question which is targeted in this paper is: What are defining attributes of exercises which have to be considered when selecting the informatics core of the exercise and the context, i.e. when defining stimuli, question, and solution?

\section{A Framework for the Design of Exercises}

Exercises and test items must be aligned to the learning objectives and to the prerequisites of course participants. Therefore, decisions about the terms or concepts in the field of Internetworking, the type of knowledge about the terms or concepts, the activity of learners, and the level of abstraction concerning the clearness of context and instruction have to be considered. These aspects can be described by (1) the content dimension, i.e. informatics core and knowledge type, (2) the process dimension, i.e. activity for solving an exercise or test item, and (3) the representation dimension, i.e. representation of stimulus, question, and answer. The attributes of an exercise considering these dimensions control the level of difficulty of an answer: The interconnectedness of concepts and the knowledge type influence the difficulty level of an exercise within the content dimension. The cognitive complexity of an activity influences the level of difficulty within the process dimension. And the level of abstraction affects the level of difficulty within the representation dimension.

Anderson et al. [6] describe Bloom's revised taxonomy for the design of assignments based upon clear definitions of learning objectives. They discern the knowledge and the cognitive process dimension. Bruner [7] describes levels of representation. These conceptions permit the assessment of assignments in terms of cognitive levels. But they abstract from the context of a subject. The author proposes the enhancement with subject specific exercise classes (EC). The development of learner adequate exercises is facilitated by the structuring of exercise classes within a taxon- 
omy which follows subject specific and didactic criteria, as well as the development of a strategy for the design of exercises from exercise classes on different levels [4].

\section{The Content Dimension}

Freischlad and Schubert describe a hierarchical classification of EC for the Didactic System Internetworking. They derived this structuring from the analysis of textbooks [5]. Each EC represents the informatics core of an exercise. Apart from previous knowledge about computer networks and information security they discern five classes at the first level which are refined at the second level. For example, the major class "Addressing" $\left(\mathrm{EC}_{3}\right)$ comprises identification of hosts within computer networks. Additionally, this class includes directory services that support information about an address. It is made-up of the sub-classes IP addressing $\left(\mathrm{EC}_{31}\right)$, Domain Name System (DNS) $\left(\mathrm{EC}_{32}\right)$, directory services $\left(\mathrm{EC}_{33}\right)$, and Network Address Translation $\left(\mathrm{EC}_{34}\right)$. As Freischlad and Schubert explain $\mathrm{EC}$ are suitable to explain the level of interconnectedness of a concrete exercise.

But to specify the content of an item we need a finer grained differentiator. Anderson et al. [6] provide such a differentiation with the knowledge dimension of the taxonomy of educational objectives. They discern factual, conceptual, procedural, and metacognitive knowledge. "These categories are assumed to lie along a continuum from concrete (Factual) to abstract (Metacognitive)" [6, p. 5]. Factual and conceptual knowledge is declarative knowledge, i.e. to "know that". Procedural knowledge is the "knowledge of how" instead of the "knowledge what". Thus, the content dimension is described by exercise classes and knowledge category.

\section{The Process Dimension}

The cognitive level is not defined by the selection of an exercise class since the concrete question or instruction defines the cognitive process. The concrete question refers to the content, i.e. the exercise class, but it is not defined by the content. Therefore, it is possible to use this category to contextualize an item. The author proposes to apply the cognitive dimension of Bloom's revised taxonomy. It comprises 19 cognitive processes assigned to six categories where the first category "Remember" focuses on retention and the following five categories focus on transfer, i.e. "Understand", "Apply", "Analyze", "Evaluate", and "Create". "The continuum underlying the cognitive process dimension is assumed to be cognitive complexity; that is, Understand is believed to be more cognitively complex than Remember, Apply is believed to be more cognitively complex than Understand, and so on" [6, p. 5].

The following example illustrates the relation between the content dimension and the process dimension. The informatics core is defined by $\mathrm{EC}_{21}$ (protocols of the application layer) and it is concretised by the selection of conceptual knowledge, i.e. the behaviour of the interacting processes. The first exercise is contextualised with the following question: "Compare the state diagrams of the Simple Mail Transfer Protocol (SMTP) and the Post Office Protocol (POP) of the server process. What is your conclusion regarding the authenticity of the client process?" The cognitive 
process is "Inferring" because the learner has to draw a logical conclusion from the information presented by the diagrams. Another exercise which is assigned to the same category within the knowledge dimension is: "Illustrate the given state diagram of an SMTP server by means of an interaction diagram which describes the transmission of an e-mail!" This exercise is assigned to the cognitive process "Exemplifying" because the learner has to find a concrete example of the principle which is given by the state diagram. Both cognitive processes are of the category Understand. Therefore this differentiation does not describe different levels of cognitive complexity.

\section{The Representation Dimension}

Bruner [7] specifies three representations which were used within the learning process. According to Bruner learners pass the levels of representation, i.e. "enactive", "iconic", and "symbolic". An example within our course is the unit about Hypertext Transfer Protocol (HTTP). The enactive representation is retrieving a web page with HTTP commands via Netcat. Learners get direct feedback whether the next step has been correct and about the reaction of the web server. An iconic representation could be an interaction diagram which illustrates the data exchange between client and server. The symbolic representation could be the formal description of HTTP. While learners have to consider syntactical correctness of the Netcat commands as well as linefeeds and whitespaces using HTTP commands on the enactive representation level they abstract from these details using interaction diagrams. At the iconic level they have to consider the data exchange between client and server for establishing a logical connection. On the symbolic level they abstract from these details focussing on the structure of HTTP commands. This example illustrates, that the modes of representation are linked to levels of abstraction. The hypothesis is that the level of difficulty increases with the level of abstraction.

Assignments of an exercise to the content category and the representation mode have to be done from a holistic point of view, i.e. considering stimulus, question, and answer. Thus, it doesn't matter whether the least abstract representation is part of the question or part of the answer. In contrast the cognitive process is determined by the separation of question and answer.

\section{Design of the Case Study}

The classroom project was performed by four teacher students during their practical course which is combined with a seminar. Furthermore the supervising teacher and the researcher attended the course with 24 students. The project spanned nine weeks and comprised seven learning units. The test was performed after eight of the nine weeks of the course and was marked as a regular test. Learners had 30 minutes to work on the test, due to the legal framework. 22 learners took part in the examination. The items were designed according to the design principle of the PISA studies [8], i.e. each of the items is composed of stimulus and related questions. Three items were selected. Each item consists of three questions. Every item was constructed with two simple questions, i.e. focusing on informatics concepts, and one question 
which combines concepts of the proceeding questions and is closely linked to a concrete phenomenon. The design was closely oriented to the learning objectives.

Within the first item learners should show if they are able to assess whether a correctly given domain name provides reliable information about the authenticity of an Internet resource which is accessed.

A1. Julia called a web page and suspects that the displayed page is not the true web page. Answer the following questions to explain if this is possible.

a) Describe the logical Internet structure by arranging the following domain names as a tree diagram representing the namespace hierarchy: www.google.de, de.wikipedia.org, en.wikipedia.org, www.die.informatik.uni-siegen.de

b) Explain the components of the domain name www.ifip.org (domain changed)!

c) Explain why in the case of DNS resolution with or without caching spoofing is not possible.

Thus, it is about the exercise class Domain Name System $\left(\mathrm{EC}_{32}\right)$. The first two questions address factual knowledge about the DNS hierarchy, i.e. the structure of the DNS namespace, and the third question addresses conceptual knowledge about the process of domain name resolving. The cognitive process category is alternating from Understand to Remember to Understand. This implies the lowest cognitive complexity of the second question. Question a) is based on an iconic representation while questions b) and c) use a symbolic representation. The first question is at the lowest level of abstraction. Thus, the third question is proposed to be at the highest level of difficulty.

The second item is about checking the authenticity of an e-mail and to assess the reliability of the result. The application e-mail is just the context in this case. The informatics concepts were represented by sub-classes of $\mathrm{EC}_{3}$ (Addressing), and $\mathrm{EC}_{4}$ (Data Transfer).

A2. Mario wants to find out the origin of an e-mail. He proposes to check an IP address. Explain if it is possible to determine the location of a host by an IP address.

a) The Internet is composed of several interconnected computer networks. Explain how they are connected and what is the functionality of the connecting component considering data exchange?

b) Insert the IP addresses 141.99 .64 .200 and 141.99 .200 .200 into the empty fields of the figure and complete the addresses in field 3 and 6. (Assign IP addresses to empty fields of a diagram representing hosts in two networks.)

c) Substantiate your proceeding for address allocation!

The questions are about conceptual knowledge. The first question addresses interconnected networks $\left(\mathrm{EC}_{52}\right)$ and packet switching on the Internet $\left(\mathrm{EC}_{42}\right)$. Learners have to name and to explain the functionality of a router. The second question asks for the connection between the components of an IP address $\left(\mathrm{EC}_{31}\right)$. And within the third question learners have to explain why and how addresses $\left(\mathrm{EC}_{31}\right)$ are assigned to one of several interconnected networks $\left(\mathrm{EC}_{52}\right)$. This assignment is a necessary prerequisite for packet switching $\left(\mathrm{EC}_{42}\right)$. Thus, the third question combines exercise classes of the other questions. The first question is assigned to the cognitive process dimension Understand. The second is assigned to Apply, because learners have to execute a familiar task. And the third question is assigned to Understand, because learners have to explain the assignment of IP addresses to different networks. Thus, the first question is at the lowest process level and the second at the highest. Fur- 
thermore, the second question is at a lower representation level (iconic) while questions a) and c) are at the same level (symbolic).

The third item is about whether learners are able to plan and process an indirect search. It focuses a specific Internet application.

A3. Julia wants to borrow a book from the school library. She decides to look in advance on the web if the book is available. Where does she have to look for the book?

a) Describe the functionality of search engines completing the given activity diagram!

b) Explain the terms web crawler and index each with two items.

c) How should Julia proceed using a search engine and when would she not succeed? Substantiate your answer!

The first question addresses conceptual knowledge about the functionality of a search engine. It is about the dynamic view on this informatics system $\left(\mathrm{EC}_{12}\right.$ : World Wide Web). The second question aimed at factual knowledge about web crawler and index which are important components of a search engine. Therefore, the static structure of the search engine is examined regarding its components $\left(\mathrm{EC}_{12}\right)$. The third question addressed procedural knowledge about search strategies based on knowledge about the functionality of a search engine $\left(\mathrm{EC}_{12}\right)$. The first question asks for an explanation of the functionality of a search engine which is assigned to the category Understand and the representation mode is iconic. The second and third questions refer to a symbolic representation. But the second is assigned to the lower process category Remember while the third question is assigned to the higher process category Apply.

\section{Findings of the Case Study}

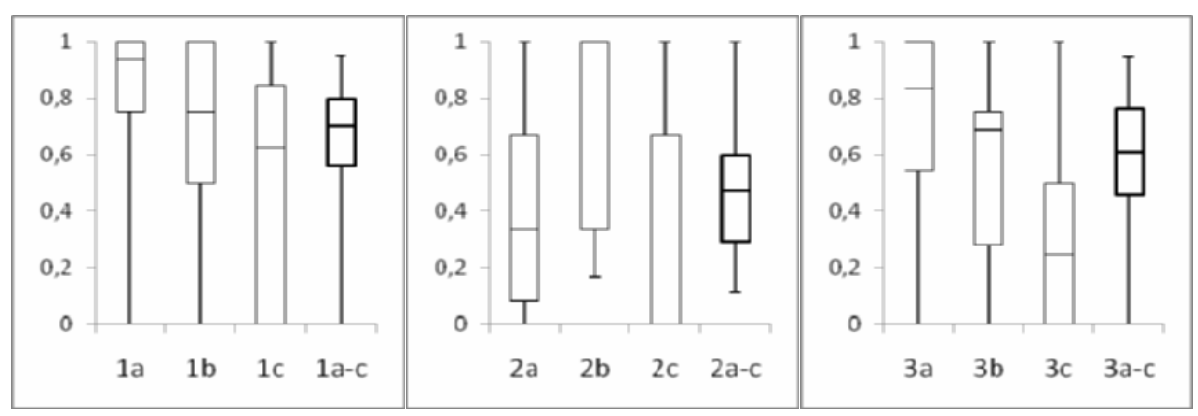

Fig. 1. Box plots of results of test items 1-3

The left diagram in Figure 1 shows the results of test item 1 relative to the total number of points for each assignment. The boxes comprise the quartiles around the median, i.e. the boxes comprise half of the results. The appended whiskers visualise the interval from maximum to minimum results. Most learners could answer these questions. Eight learners could not answer question 1c. But there were four learners who achieved the full amount of points. The middle diagram shows the results of test item 2. While less than half of the learners could answer the first question sufficiently, i.e. they have got more than half of the points, the second question was an- 
swered completely correct by 12 learners. The third question was answered correctly by four learners. But the median is 0 points. Therefore the median of item 2 is at 0.47. The right diagram shows the results of test item 3. Three-fourths could complete the activity diagram with more than 50 per cent of the points. Question two could be answered by most learners with more than half of the points. But the third question was answered by three-fourths in a way that they achieved less than half of the points. Just four learners could apply indirect search to the described situation.

The results are discussed considering the assignment of the questions within the framework. The framework does not provide a weighting of the three dimensions regarding the level of difficulty. The results of the test are interpreted regarding the influence of the dimensions to the level of difficulty. Just the questions of a single test item were compared to avoid effects related to the prerequisites of the learners regarding the differing contents. In Table 1 the assignment of the items according to the predefined framework is shown. As far as applicable within parentheses the level of the attribute within the dimension is shown.

Table 1. Test items in the framework

\begin{tabular}{lllll}
\hline \multicolumn{2}{l}{ Test Item } & Content & Process & Representation \\
\hline $\mathrm{A} 1$ & $\mathrm{a}$ & $\mathrm{EC}_{32} /$ Factual (0) & Understand (1) & iconic (1) \\
& $\mathrm{b}$ & $\mathrm{EC}_{32} /$ Factual (0) & Remember (0) & symbolic (2) \\
& $\mathrm{c}$ & $\mathrm{EC}_{32} /$ Conceptual (1) & Understand (1) & symbolic (2) \\
$\mathrm{A} 2$ & $\mathrm{a}$ & $\mathrm{EC}_{42}, \mathrm{EC}_{52} /$ Conceptual (1) & Understand (1) & symbolic (2) \\
& $\mathrm{b}$ & $\mathrm{EC}_{31} /$ Conceptual (1) & Apply (2) & iconic (1) \\
& $\mathrm{c}$ & $\mathrm{EC}_{31}, \mathrm{EC}_{42}$, EC \\
52 & $/$ Conceptual (1) & Understand (1) & symbolic (2) \\
$\mathrm{A} 3$ & $\mathrm{a}$ & $\mathrm{EC}_{12} /$ Conceptual (1) & Understand (1) & iconic (1) \\
& $\mathrm{b}$ & $\mathrm{EC}_{12} /$ Factual (0) & Remember (0) & symbolic (2) \\
& $\mathrm{c}$ & $\mathrm{EC}_{12} /$ Procedural (2) & Apply (2) & symbolic (2) \\
\hline
\end{tabular}

The results of A1 indicate that the representation mode strongly influences the level of difficulty. Although question b) would be assigned to a lower level of difficulty according to the cognitive process question a) is answered better. The explanation which is derived of the framework indicates that the iconic representation of question a) and therefore the lower level of abstraction is the reason for better results. Furthermore, the comparison of the questions b) and c) indicate that the knowledge category and the cognitive process category affect the level of difficulty. The comparison of the question A2 a) and b) do not allow conclusions because they are items of different exercise classes. But the interconnectedness of exercise classes in question c) (compared to a)) respectively in combination with the representation mode (compared to b)) results in a higher level of difficulty. The results of A3 indicate that the representation mode mostly affects the level of difficulty because question a) is assigned at a higher level of difficulty according to the content and the process dimension than question b). Nevertheless the results are better. Furthermore the effect of the content in combination with the process dimension to the level of difficulty is confirmed comparing question $b$ ) and $c$ ).

The results indicate that the level of difficulty is varied by the modes of representation and the combination of content and process dimension. Thus, the framework is 
applicable to explain levels of difficulty. Whilst the content category and the cognitive process are derived from the learning objectives which are used for the design of the learning process, the representation mode is independently chosen to vary the level of difficulty. This knowledge can be used when creating new or modify exercises respectively test items.

\section{Conclusion}

The aim of this framework is to enable teachers respectively teacher students and teacher novices to design high quality items in the field of Internetworking. Attributes which affect the level of difficulty are defined. The content, process, and representation dimension are appropriate to describe the design strategy of exercises and test items. The results of the case study indicate that the design of exercises can be aligned to this framework. The framework has to be validated in further empirical studies with more items so that the distinction of the attributes for the evaluation becomes clearer. And the framework has to be applied in teacher education to affirm its feasibility. In 2008 we will conduct practical training in school with teacher students. Thus, we will get feedback to this approach.

\section{References}

1. Tucker, A. (ed.): A model curriculum for $\mathrm{K}-12$ computer science: final report of the ACM K-12 task force curriculum committee. ACM, New York 2006.

URL: http://www.csta.acm.org/Curriculum/sub/CurrFiles/K-12ModelCurr2ndEd.pdf (January 2008)

2. Anderson, J.; van Weert, T. (eds.): Information and communication technology in secondary education - A Curriculum for Schools. UNESCO, Paris 2002.

URL: http://wwwedu.ge.ch/cptic/prospective/projets/unesco/en/curriculum2000.pdf (January 2008)

3. Freischlad, S.: Learning Media Competences in Informatics. In: Dagiene, V.; Mittermeir, R. (eds.): Proceedings of Second International Conference on "Informatics in Secondary Schools. Evolution and Perspectives - ISSEP”, November 7-11, Vilnius (Lithuania) 2006, pp. 591-599, ISBN 9955-680-47-4.

4. Brinda, T.: Development of the exercise culture in informatics. In: [9]

5. Freischlad, S.; Schubert, S.: Towards High Quality Exercise Classes for Internetworking. In: [9]

6. Anderson, L. W.; Krathwohl, D. R. (eds.): A taxonomy for learning, teaching and assessing: A revision of Bloom's Taxonomy of educational objectives. Addison Wesley Longman, New York 2001.

7. Bruner, J. S.; Olver, R. R.; Greenfield, P. M.: Studies in Cognitive Growth. John Wiley, New York 1966.

8. OECD: Learning for Tomorrow's World - First Results from PISA 2003. Organisation for Economic Co-operation and Development, Paris 2004.

URL: http://www.pisa.oecd.org/dataoecd/1/60/34002216.pdf (January 2008)

9. Benzie, D.; Iding, M. (eds.): Proceedings of IFIP-Conference on "Informatics, Mathematics and ICT: A golden triangle", June 27-29, Boston (USA) 2007, ISBN-13: 978-0-61514623-2. 